\title{
Memory-assisted decoder for approximate Gottesman-Kitaev-Preskill codes
}

\author{
Kwok Ho Wan, ${ }^{1,2, *}$ Alex Neville $\odot,{ }^{2}$ and Steve Kolthammer ${ }^{2}$ \\ ${ }^{1}$ Mathematical Physics, Department of Mathematics, Imperial College London, London SW7 2AZ, United Kingdom \\ ${ }^{2}$ QOLS, Blackett Laboratory, Imperial College London, London SW7 2AZ, United Kingdom
}

(Received 11 February 2020; revised 17 August 2020; accepted 18 August 2020; published 24 November 2020)

\begin{abstract}
We propose a quantum error correction protocol for continuous-variable finite-energy, approximate Gottesman-Kitaev-Preskill (GKP) states undergoing small Gaussian random displacement errors, based on the scheme of Glancy and Knill [Phys. Rev. A 73, 012325 (2006)]. We show that combining multiple rounds of error-syndrome extraction with Bayesian estimation offers enhanced protection of GKP-encoded qubits over comparible single-round approaches. Furthermore, we show that the expected total displacement error incurred in multiple rounds of error followed by syndrome extraction is bounded by $2 \sqrt{\pi}$. By recompiling the syndrome-extraction circuits, we show that all squeezing operations can be subsumed into auxiliary state preparation, reducing them to beamsplitter transformations and quadrature measurements.
\end{abstract}

DOI: 10.1103/PhysRevResearch.2.043280

\section{INTRODUCTION}

Encoding and manipulating quantum information in continuous variable $(\mathrm{CV})$ systems [1-3] is a promising route to realizing a useful quantum computing device. Large-scale CV cluster states can be generated on demand [4], and fast, high-quality one- and two-qubit Clifford gates are deterministically available [5]. Fault-tolerant, measurement-based quantum computation is possible using CV cluster states along with CV measurements and non-Gaussian state injection [6,7], though the levels of squeezing required for fault tolerance are beyond the reach of current experiments $[6,8-$ $11]$.

A leading approach to $\mathrm{CV}$ quantum computation, proposed by Gottesman, Kitaev, and Preskill (GKP) [12], is based on the idea of encoding a qubit within an (infinite dimensional) oscillator. Ideal codeword wave functions within this paradigm correspond to infinite-energy Dirac combs and are commonly referred to as GKP states. In practice, this ideal wave function is replaced by a finite-energy approximation, such as a comb of narrow Gaussian peaks modulated by a broad Gaussian envelope.

The appeal of GKP-encoded qubits is that they possess both an intrinsic robustness to physically motivated error channels and natural schemes for error-syndrome extraction and correction. An initial proposal for GKP error correction was based on CV stabilizer generalizations of the Steane circuits [13] for error-syndrome extraction. Subsequently, Glancy and Knill proposed a different method based on a

\footnotetext{
${ }^{*}$ Corresponding author: kwok.wan14@imperial.ac.uk

Published by the American Physical Society under the terms of the Creative Commons Attribution 4.0 International license. Further distribution of this work must maintain attribution to the author(s) and the published article's title, journal citation, and DOI.
}

beamsplitter transformation [14], which provides the basis for the scheme detailed in this paper.

We define approximate GKP codewords with width $\vec{\Delta}=$ $(\Delta, \kappa)$ as

$$
\psi_{\mu}^{\vec{\Delta}}(x) \propto \sum_{s \in \mathbb{Z}} G_{\frac{1}{k}}[(2 s+\mu) \sqrt{\pi}] G_{\Delta}[x-(2 s+\mu) \sqrt{\pi}],
$$

where $G_{\Sigma}(z)=\exp \left(-\frac{z^{2}}{2 \Sigma^{2}}\right)$ and $\mu \in\{0,1\}$ defines the logical basis states. Informally, for the logical 0 (1) state we have a superposition of Gaussians of width $\Delta$ centered at even (odd) values of $\sqrt{\pi}$, with an overall Gaussian envelope of width $1 / \kappa$. Better approximations to the ideal GKP state are achieved with smaller values of $\Delta$ and $\kappa$, although these also correspond to larger average energy and an apparent increase in experimental difficulty.

Recently, Albert et al. [15] showed that the GKP code outperforms a number of other bosonic codes when states are exposed to amplitude damping and Gaussian random displacement errors. We consider the latter and write such an error acting on the state $\hat{\rho}$ as

$$
\mathcal{E}_{\sigma_{0}}(\hat{\rho})=\frac{1}{2 \pi \sigma_{0}^{2}} \iint_{\alpha \in \mathbb{C}} d^{2} \alpha G_{\sigma_{0}}(|\alpha|) \hat{D}(\alpha) \hat{\rho} \hat{D}(\alpha)^{\dagger},
$$

where the operator $\hat{D}(\alpha)$ shifts the state in phase space by $\operatorname{Re}\{\alpha\}, \operatorname{Im}\{\alpha\}$ in the $q$ and $p$ quadratures, respectively, and the width $\sigma_{0}$ quantifies the extent of the error. Notably, this error model describes amplitude damping that is preceded by an offsetting preamplification [16], and it is therefore highly relevant to many experimental platforms. Despite this potential, explicit error correction protocols for accessible approximate states are currently lacking.

In this article we present a decoder for the GKP code undergoing Gaussian random displacement errors, based on the Glancy and Knill [14] error recovery scheme and Bayesian estimation. In particular, we extend the scheme to enable enhanced error estimation using multiple syndrome extractions, 


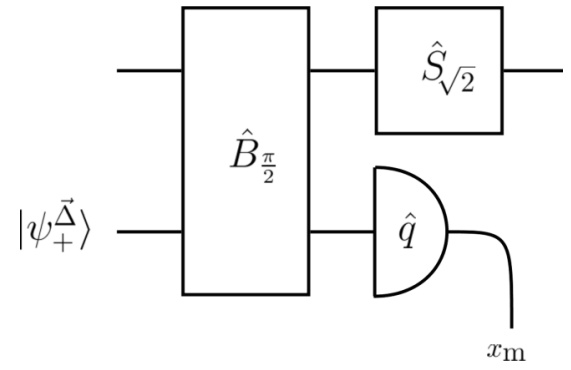

FIG. 1. The $q$-quadrature error-syndrome extraction circuit ( $q$ $\mathrm{SE}$ ), as proposed by Glancy and Knill [14]. The GKP qubit and an auxiliary state are input in the top and bottom modes, respectively. Following beamsplitting and squeezing operations, the error syndrome $x_{m}$ is generated by a $q$-quadrature measurement on the auxiliary mode.

enabling improved error supression which we show to be useful in extending the lifetime of states with a mean number of bosons as low as ten. We find that many rounds of syndrome extraction without active corrective displacement causes the qubit to drift in phase space by at most approximately $2 \sqrt{\pi}$ in each quadrature. Additionally, we recompile the syndrome extraction circuit in an experimentally friendly way, such that squeezing need only be applied to auxiliary states which can be prepared offline.

\section{SYNDROME EXTRACTION}

The GKP syndrome extraction scheme of Glancy and Knill [14] can be broken down into two sequential circuits: $q-\mathrm{SE}$ and $p$-SE, which extract the error syndromes in the $q$ and $p$ quadratures, respectively (see Fig. 1).

We begin our analysis with an arbitrary input qubit wave function $Q^{\vec{\Delta}}(x)$ that has undergone an unknown displacement error $(u, v)$ :

$$
Q^{\vec{\Delta}}(x) \underset{(u, v)}{\stackrel{\text { error }}{\longrightarrow}} e^{i v x} Q^{\vec{\Delta}}(x-u) .
$$

This corrupted qubit is input into the top mode of the $q$-SE circuit, while an auxiliary GKP state, $\left|\psi_{+}^{\vec{\Delta}}\right\rangle \propto\left|\psi_{0}^{\vec{\Delta}}\right\rangle+\left|\psi_{1}^{\vec{\Delta}}\right\rangle$, is input into the bottom mode. The action of the $q$-SE circuit can be visualized in terms of the two-mode $q$-quadrature wave function. The beamsplitter $\hat{B}_{\frac{\pi}{2}}$ causes an anticlockwise rotation by $45^{\circ}$ in the joint quadrature space, and the subsequent squeezer $\hat{S}_{\sqrt{2}}$ scales the top-mode quadrature by $\sqrt{2}$. The error syndrome is then generated by a $q$-quadrature measurement of the auxiliary mode.

The $p$-SE circuit proceeds similarly, although there are subtle differences beyond a change of variables $q \rightarrow p$ and squeezing in the conjugate direction $\left(\hat{S}_{\sqrt{2}} \rightarrow \hat{S}_{\sqrt{2}}^{\dagger}\right)$. Indeed, we must also change the auxiliary state $\left|\psi_{+}^{\vec{\Delta}}\right\rangle \rightarrow\left|\psi_{0}^{\vec{\Delta}^{\prime}}\right\rangle$, where $\vec{\Delta}^{\prime}=(\Delta / \sqrt{2}, \kappa \sqrt{2})$ - a departure from the proposal of Glancy and Knill, which we found necessary for sequential $q$-SE and $p$-SE circuits to be applied to approximate GKP states (see Appendix B for more details).
Following sequential $q$-SE and $p$-SE circuits, the qubit has transformed according to

$$
e^{i v x} Q^{\vec{\Delta}}(x-u) \underset{\mathrm{SE}}{\stackrel{q-, p-}{\longrightarrow}} e^{i \theta\left(p_{m}, v\right) x} Q^{\vec{\Delta}}\left[x-\theta\left(x_{\mathrm{m}}, u\right)\right],
$$

with a high fidelity (see Appendix D) when the displacement is small. Here, $\theta\left(x_{m}, u\right)$ is given by

$$
\theta\left(x_{\mathrm{m}}, u\right)=\frac{u}{2}-f_{\text {step }}^{*}\left(x_{m}\right),
$$

where $f_{\text {step }}^{*}\left(x_{m}\right)$ is the modified modular division by 4 function defined in Appendix $\mathrm{A}$, and $\theta\left(p_{m}, v\right)$ is defined similarly. These quantities can be interpreted as the total displacement experienced by the qubit: an unknown, random part, $\frac{u}{2}$, remaining from the error channel and a known part, $f_{\text {step }}^{*}\left(x_{m}\right)$, introduced by the measurement.

While an error-correcting procedure could involve estimation of the total displacement $\theta\left(x_{m}, u\right)$ and the immediate application of a corrective displacement based on this estimate, we note that allowing an uncorrected qubit to undergo a further syndrome extraction process only results in another displaced version of the input qubit. With this in mind, our decoder uses measurement information from multiple rounds of errors and syndrome extraction without intermediate correction to estimate a single corrective displacement to apply. This memory-assisted approach is summarized in Fig. 2, alongside a contrasting memoryless approach which applies a correction after each syndrome extraction and then forgets about it.

We now proceed by extending our analysis from one round to multiple rounds of syndrome extraction. Say that after the $h$ th round of syndrome extraction we have histories of $q$-quadrature syndrome measurements $\vec{x}_{m}=\left(x_{m}^{(1)}, \ldots, x_{m}^{(h)}\right)$ and displacement errors $\vec{u}=\left(u_{1}, \ldots, u_{h}\right)$, and similarly $p$ quadrature values $\vec{p}_{m}=\left(p_{m}^{(1)}, \ldots, p_{m}^{(h)}\right)$ and $\vec{v}=\left(v_{1}, \ldots, v_{h}\right)$ for the $p$-quadrature. If each displacement error is small, then the input qubit is transformed according to

$$
Q^{\vec{\Delta}}(x) \rightarrow e^{i \theta_{h}\left(\vec{p}_{m}, \vec{v}\right) x} Q^{\vec{\Delta}}\left[x-\theta_{h}\left(\vec{x}_{m}, \vec{u}\right)\right] .
$$

with high fidelity (again, see Appendix D). Focusing on the $q$ quadrature: the total shift in $q$ is given by

$$
\begin{aligned}
\theta_{h}\left(\vec{x}_{m}, \vec{u}\right) & =\sum_{j=1}^{h}\left[\frac{u_{j}}{2^{h-j+1}}\right]-\sum_{k=1}^{h}\left[\frac{f_{\text {step }}^{*}\left[\mathcal{X}_{m}^{(k)}\left(\vec{x}_{m}\right)\right]}{2^{h-k}}\right] \\
& =\theta_{h}^{\operatorname{err}}(\vec{u})-\theta_{h}^{\text {step }}\left(\vec{x}_{m}\right),
\end{aligned}
$$

where $\mathcal{X}_{m}^{(k)}$ can be interpreted as the measurement outcome $x_{m}^{(k)}$ transformed to account for previous measurementinduced shifts and is given explicitly by

$$
\mathcal{X}_{m}^{(h)}\left(\vec{x}_{m}\right)=x_{m}^{(h)}+\frac{1}{\sqrt{2}} \sum_{j=1}^{k-1}\left[\frac{f_{\text {step }}^{*}\left[\mathcal{X}_{m}^{(j)}\left(\vec{x}_{m}\right)\right]}{2^{k-j-1}}\right]
$$

for $h>1$, and $\mathcal{X}_{m}^{(1)}=x_{m}^{(1)}$. In analogy with the single-round case above, we have an unknown contribution to the total displacement $\theta_{h}^{\text {err }}(\vec{u})$ together with the known, measurementinduced contribution $\theta_{h}^{\text {step }}\left(\vec{x}_{m}\right)$.

Without active corrective shifts at each round, the distance a GKP qubit drifts in phase space after $h$ rounds is 


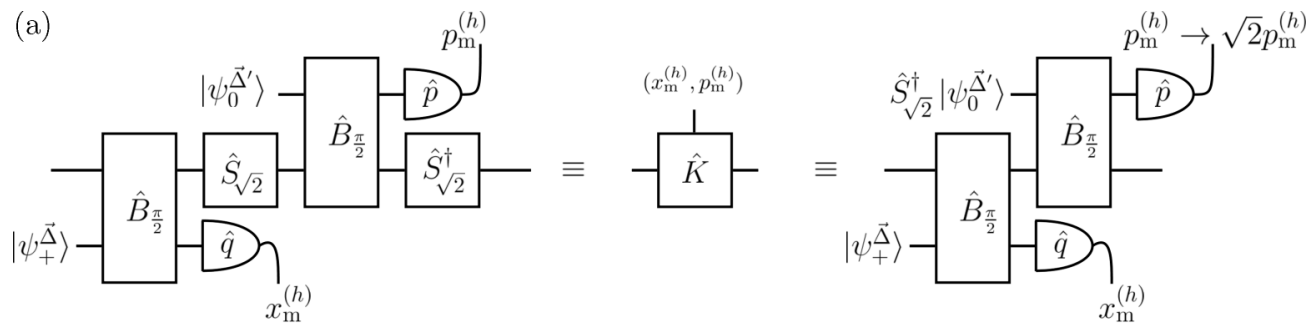

(b)

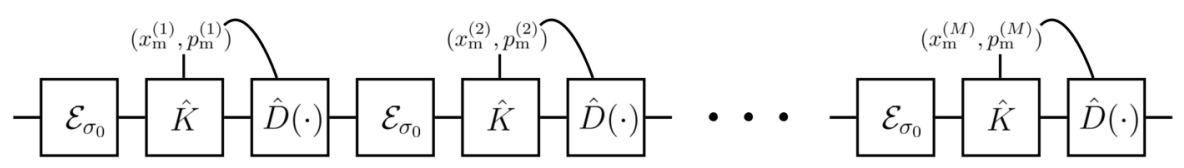

(c)

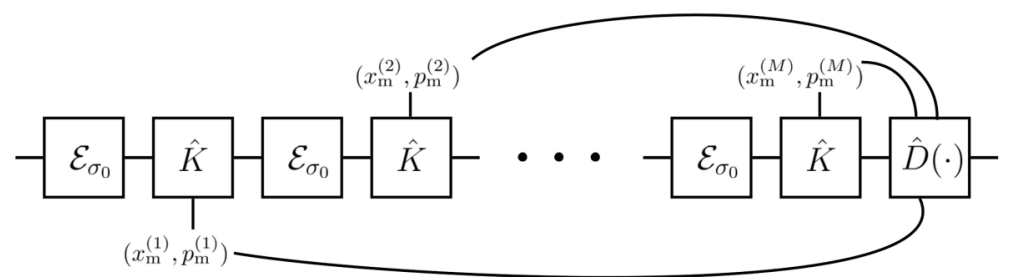

FIG. 2. (a) The standard $q$ - and $p$-quadrature syndrome extraction circuits (left), which feature squeezing operations on the qubit mode, can be represented by a measurement-dependent Kraus operator $\hat{K}$ (center), and recompiled such that squeezing is applied to the $p$-auxiliary state, with a reinterpreted $p$-quadrature measurement (right). (b) Memoryless error correction: Syndrome measurement results are used to inform a corrective displacement at each round, and no information is carried forward to future rounds. (c) Memory-assisted error correction: No active corrective shift is performed after each syndrome measurement. Instead, all the syndrome measurement results are used together to decode and perform a single corrective displacement after $M$ rounds.

bounded above as $\left|\theta\left(\vec{x}_{m}, \vec{u}\right)\right|<2 \sqrt{\pi}\left(1-2^{-h}\right)+|X|$, where $X \sim \mathcal{N}\left(0, \sigma_{0}^{2} \frac{\left(1-4^{-h}\right)}{3}\right)$ is a Gaussian random variable with mean 0 and variance $\sigma_{0}^{2} \frac{\left(1-4^{-h}\right)}{3}$ (see Appendix $C$ for proof). It follows that the expected value for total displacement error in each quadrature after any number of syndrome extraction rounds is bounded above by approximately $2 \sqrt{\pi}$.

In Fig. 2(a), we identify a practical simplification to the combined $q$-SE and $p$-SE circuit that moves all squeezing operations offline to auxiliary state preparation. To do so, we use a modified $p$-SE auxiliary state, $\hat{S}_{\sqrt{2}}^{\dagger}\left|\psi_{0}^{\vec{\Delta}^{\prime}}\right\rangle$, and reinterpret the $p$-quadrature measurement $\left(p_{\mathrm{m}} \rightarrow \sqrt{2} p_{\mathrm{m}}\right)$ (see Appendix $\mathrm{F}$ for proof).

\section{DECODER (BAYESIAN ESTIMATION)}

We now show how to use the $q$ - and $p$-SE measurement outcomes to estimate the final displacements when the loss channel is well characterized (i.e., $\sigma_{0}$ is known). For convenience, we set $\kappa=\Delta$ in this section, although it is straightforward to extend the results to the more general case.

In the case of a single round of $q$-SE and $p$-SE application, the probability of measuring $x_{m}$ given a shift error $u$ can be approximated as $\mathbb{P}\left(x_{m} \mid u\right) \propto \psi_{+}^{\vec{\Delta}}\left(\sqrt{2} x_{m}-u\right)$, which we note is independent of both $v$ and $p_{m}$. By using a prior probability density function (PDF) corresponding to our characterized error channel and applying Bayes' theorem, we obtain a posterior PDF:

$$
\mathbb{P}\left(u \mid x_{m}\right) \propto \psi_{+}^{\vec{\Delta}}\left(\sqrt{2} x_{m}-u\right) e^{-\frac{u^{2}}{2 \sigma_{0}^{2}}} .
$$

Assuming that $\vec{\Delta}$ and $\sigma_{0}$ are both small compared to $\sqrt{\pi}$, this posterior is well approximated by

$$
\mathbb{P}\left(u \mid x_{m}\right) \approx \mathcal{N}\left(\frac{\sqrt{2} \sigma_{0}^{2} x_{m}-\sqrt{\pi} \sigma_{0}^{2}\left\lfloor\frac{\sqrt{2} x_{m}}{\sqrt{\pi}}\right\rceil}{\Delta^{2}+\sigma_{0}^{2}}, \frac{\Delta^{2} \sigma_{0}^{2}}{\Delta^{2}+\sigma_{0}^{2}}\right),
$$

and we take our estimate $\tilde{u}$ of the shift error $u$ to be the mean of this Gaussian. Explicitly,

$$
\tilde{u}=\frac{\sqrt{2} \sigma_{0}^{2} x_{m}-\sqrt{\pi} \sigma_{0}^{2}\left\lfloor\frac{\sqrt{2} x_{m}}{\sqrt{\pi}}\right\rceil}{\Delta^{2}+\sigma_{0}^{2}},
$$

which is a good approximation of the minimum mean square error (MMSE) estimator for $u$. The estimate for the displacement required to counteract the acquired error is therefore $\theta\left(x_{m}, \tilde{u}\right)$, from combining Eqs. (5) and (11).

The corresponding $p$-quadrature conditional probabilities can be obtained by substituting $\left(u, x_{m}, \Delta\right) \rightarrow\left(v, p_{m}, 2 \Delta\right)$, where the asymmetry between the $q$ quadrature and the $p$ quadrature originates from the differing widths of the auxiliary states.

For multiple rounds, the estimation of the cumulative unknown displacement caused by the vector $\vec{u}$ is achieved in the same spirit as estimating the parameter $u$ in the single-round 
case, except with a messier-looking (although, still efficiently computable) estimator.

The probability of obtaining the $q$-quadrature measurement outcome $x_{m}^{(h)}$ in the $h$ th round, given the measurement values obtained in the previous $(h-1)$ rounds and displacement shifts in all $h$ rounds, is $\mathbb{P}\left(x_{m}^{(h)} \mid u_{1}, \ldots, u_{h}, x_{m}^{(1)}, \ldots, x_{m}^{(h-1)}\right) \propto$ $\psi_{+}^{\vec{\Delta}}\left(\sqrt{2} x_{\mathrm{m}}^{(h)}-\mathcal{U}_{h}\right)$. Here, $\mathcal{U}_{h}$ is the shift contributed in the $h$ th round by the error channel, transformed to account for all previous steps (see Appendix A for an explicit definition). We now apply Bayes' theorem with Gaussian prior PDFs for the error in each of the $M$ rounds, resulting in the posterior PDF

$$
\mathbb{P}_{M}^{(q)}\left(\vec{u} \mid \vec{x}_{m}\right) \propto \prod_{h=1}^{M} \psi_{+}^{\vec{\Delta}}\left(\sqrt{2} x_{m}^{(h)}-\mathcal{U}_{h}\right) G_{\sigma_{0}}\left(u_{h}\right),
$$

Assuming, again, that $\sigma_{0}$ and $\Delta$ are small compared to $\sqrt{\pi}$, we show that the posterior $\mathbb{P}_{M}^{(\mathrm{q})}\left(\vec{u} \mid \vec{x}_{m}\right)$ is well approximated by a multivariate Gaussian $\mathcal{N}(\overrightarrow{\tilde{u}}, \Sigma)$ with the mean vector $\overrightarrow{\tilde{u}}$ and the covariance matrix $\Sigma$, in analogy with the single-round case. The quantity that we actually wish to estimate is $\theta_{M}^{\operatorname{err}}(\vec{u})$ from Eq. (7), which is a linear combination of elements from $\vec{u}$. The corresponding PDF is given by

$$
\mathbb{P}\left(\theta_{M}^{\operatorname{err}}(\vec{u}) \mid \vec{x}_{m}\right) \approx \mathcal{N}\left(\vec{a} \cdot \overrightarrow{\tilde{u}}, \vec{a}^{\mathrm{T}} \cdot \Sigma \cdot \vec{a}\right),
$$

with $a_{k}=2^{-(M+1-k)}$ and

$$
\begin{aligned}
\tilde{u}_{k}= & \left(\frac{\sigma_{0}}{\Delta}\right)^{2}\left\{2^{k} \sum_{j=1}^{M} \frac{F_{j}}{2^{j}}-\left(\frac{\sigma_{0}}{\Delta}\right)^{2} \sum_{h=1}^{M}\right. \\
& \left.\times\left[\left(\sum_{n=m_{k, h}}^{M} \frac{2^{k+h}}{4^{n}}\right)\left(\sum_{j=h}^{M} \frac{F_{j}}{2^{j}}\right)\right] 2^{h}\right\},
\end{aligned}
$$

to order $\left(\frac{\sigma_{0}}{\Delta}\right)^{4}$, where $m_{k, h}=\max \{k, h\}$ and $F_{h}=\sqrt{2} \mathcal{X}_{m}^{(h)}-$ $\sqrt{\pi}\left\lfloor\sqrt{2} \mathcal{X}_{m}^{(h)} / \sqrt{\pi}\right\rceil$.

As in the single-round case, the posterior mean is used as the MMSE estimator for the displacement error. We see that the posterior is well approximated by a Gaussian with a mean $\vec{a} \cdot \overrightarrow{\tilde{u}}$ which can be calculated directly from syndrome measurement results. An explicit approach to this calculation is presented as an algorithm in Appendix E.

Also of interest is the variance of this Gaussian, $\vec{a}^{\mathrm{T}} \cdot \Sigma$. $\vec{a}$, as it corresponds to the uncertainty in our estimation. In Appendix $\mathrm{C}$ we show that, if $\frac{\sigma_{0}}{\Delta}<\frac{1}{2}$ (which is true for all $M>1$ ), the variance converges to

$$
\begin{aligned}
V_{\mathrm{q}}(M)= & \frac{\sigma_{0}^{2}}{3}\left\{\left(1-4^{-M}\right)+\left(\frac{\sigma_{0}}{\Delta}\right)^{2} \frac{4}{9}\left[4^{-2 M}\right.\right. \\
& \left.\left.+3(1+2 M) 4^{-M}-4\right]\right\},
\end{aligned}
$$

neglecting terms within the bracket of order $\left(\frac{\sigma_{0}}{\Delta}\right)^{4}$ and higher. Note that $V_{q} \rightarrow \frac{\sigma_{0}^{2}}{3}$ very quickly as $M$ grows, given that $\frac{\sigma_{0}}{\Delta} \ll$ 1.

\section{NUMERICAL RESULTS}

The aim of a quantum error correction (QEC) procedure is to protect logical quantum information. Pantaleoni et al.

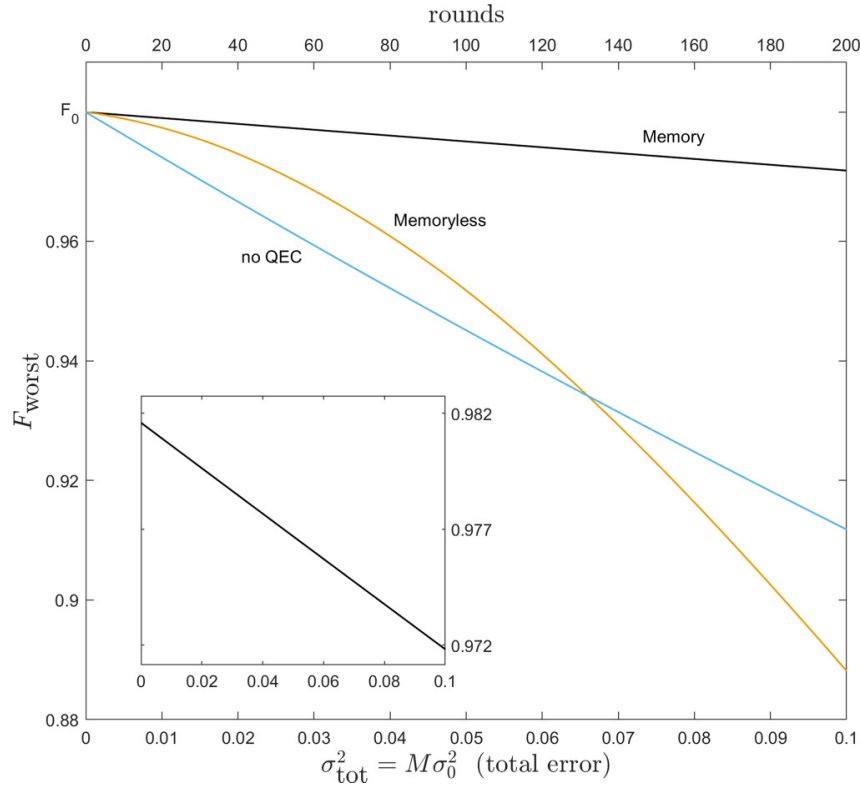

FIG. 3. Qubit fidelity achieved by the memory-assisted decoder (black line), the memoryless decoder (yellow line), and no QEC (aqua line). A Gaussian error channel of width $\sigma_{0}^{2}=0.0005$ is applied in each of the $M$ rounds of syndrome extraction to a GKP state with width $\Delta=\kappa=0.22$. The initial fidelity $F_{0}=0.981$, and the inset plot shows how the black curve decreases over a smaller range.

recently reported a method to extract logical information from approximate GKP states [17]. In essence, this reduces a density matrix function describing a $\mathrm{CV}$ state to a qubit density matrix $\left(\hat{\rho}^{\mathrm{CV}} \rightarrow \hat{\rho}^{\text {qubit }}\right)$. Using this, fidelities between qubit density matrices at the input and output of an error correction procedure can be computed in order to benchmark its performance.

In Fig. 3 we present the results of numerical simulations for the specific case of a GKP code with $\Delta \approx 0.22$ (corresponding to an average number of bosons $\bar{n} \approx 10$ ). For varying numbers of rounds of error channel application followed by syndrome extraction, we benchmark error correction with our memory-assisted decoder against density matrix function simulations of the memoryless decoder and no error correction at all.

We observe that error correction is improved by using our memory-assisted decoder instead of the memoryless decoder-a finding similar to that of Vuillot et al. [18] and Noh and Chamberland [9] for Steane-based GKP syndrome extraction. Note that $\Delta$ is large enough that the memoryless decoder performs worse than no QEC once a few hundred rounds are considered. We also see that the quality of the memory-assisted error-corrected state is higher than that of the uncorrected state, despite the state containing a low average number of bosons. Furthermore, the fidelity of the input state is approximately preserved through application of the memory-assisted error-correction scheme.

\section{SUMMARY AND OUTLOOK}

We have described an explicit protocol for GKP quantum error correction that provides improved protection from 
Gaussian displacement errors with reduced experimental requirements. Notably, we have shown that GKP states undergo a total displacement (and therefore require a correction) that is approximately bounded by $2 \sqrt{\pi}$ in each quadrature, after multiple rounds of error-syndrome extraction. A memoryassisted decoder based on Bayesian estimation was developed to specify the near-optimal corrective displacement, and numerical simulations have shown that this results in significantly improved error correction compared to a memoryless decoder when applied to an approximate GKP state with a small average number of bosons.

Recent experiments have demonstrated GKP states encoded in oscillations of trapped ions [10] and microwave fields of superconducting resonators [11]. In the latter case, states are shown with widths of $(\Delta, \kappa)=(0.16,0.32)$. As these values are similar to those we have studied here, this suggests that our decoder can be exploited by near-term experiments as part of an error correction procedure. Additionally, the GlancyKnill syndrome extraction circuit at the core of our method may be better suited to realistic devices than the more studied SUM gate syndrome extraction, since the latter requires more gates, and therefore more physical components, to implement [19].

The error model that we have considered in this work also pertains to errors that arise in teleporting a GKP state along a CV cluster state with finite squeezing [20]. In this situation, the finite squeezing of the cluster state induces a displacement error. We anticipate that it will therefore be possible to apply our error correction scheme between nodes on a $\mathrm{CV}$ cluster state graph and thereby lower the squeezing threshold for universal fault-tolerant quantum computation using $\mathrm{CV}$ cluster states and GKP state injection.

\section{DATA AVAILABILITY STATEMENT}

This is a theoretical paper and there are no experimental data available beyond the numerical simulation data described in the paper. K.H.W. performed all the numerical experiments, all the authors contributed to the manuscript, and the project was supervised by A.N. and S.K.

\section{ACKNOWLEDGMENTS}

We acknowledge discussions with Luca Cocconi, Jacob Hastrup, Hlér Kristjánsson, Y. H. Chang, Frederic Sauvage, Jonathan Conrad, Christophe Vuillot, Kyungjoo Noh, Giacomo Pantaleoni, Myungshik Kim, and Hyukjoon Kwon. K.H.W. is funded by the President's Ph.D. Scholarship of Imperial College London. S.K. acknowledges support from EPSRC Grant No. EP/T001062/1.

\section{APPENDIX A: NOTATION AND DEFINITIONS}

Throughout our text, we use the following notations and conventions:

(i) Plank's constant $\hbar=1$.

(ii) Displacement operator $\hat{D}(\alpha)=e^{\frac{\alpha \hat{a}^{\dagger}-\alpha^{*} \hat{a}}{\sqrt{2}}}$.

(iii) Beamsplitter operator $\hat{B}(\phi)=e^{-\frac{\phi}{2}\left(\hat{a}^{\dagger} \hat{b}-\hat{a} \hat{b}^{\dagger}\right)}$.

(iv) Squeezing operator $\hat{S}_{q}(a)=e^{\frac{\ln (a)}{2}\left[\hat{a}^{2}-\left(\hat{a}^{\dagger}\right)^{2}\right]}$. (v) Gaussian function $G_{\sigma}(x)=\exp \left(-\frac{x^{2}}{2 \sigma^{2}}\right)$.

(vi) $\mathcal{N}(\mu, V)$ is a Gaussian random vector with mean $\mu$ and covariance $V$.

(vii) The Rounding function $\lfloor x\rceil$ gives the nearest integer to $x$.

(viii) Remainder function $\operatorname{rem}(n, 4)$ gives the remainder from dividing integer $n$ by 4 .

Additionally, we define the following:

(i) GKP wave function widths are $\vec{\Delta}=(\Delta, \kappa)$ and $\vec{\Delta}^{\prime}=$ $(\Delta / \sqrt{2}, \kappa \sqrt{2})$.

(ii) Normalized GKP wave functions are defined as

$\psi_{\mu}^{\vec{\Delta}}(x)=N_{\mu} \sum_{s \in \mathbb{Z}} G_{\frac{1}{\kappa}}[(2 s+\mu) \sqrt{\pi}] G_{\Delta}[x-(2 s+\mu) \sqrt{\pi}]$.

(iii) Step functions are defined as

$$
\begin{aligned}
s(x) & =\frac{1}{2} \operatorname{rem}(\lfloor x / \sqrt{\pi / 2\rceil}, 4), \\
f_{\text {step }}^{*}(x) & =\frac{\sqrt{\pi}}{2} \operatorname{rem}^{*}(\lfloor x / \sqrt{\pi / 2}\rceil, 4) .
\end{aligned}
$$

(vi) The effective measurement results $\mathcal{X}_{\mathrm{m}}^{(h)}$ and the effective errors $\mathcal{U}_{h}$ if passive error correction is carried out are as follows:

$$
\begin{aligned}
& \mathcal{X}_{m}^{(h)}=x_{m}^{(h)}+\frac{1}{\sqrt{2}} \sum_{k=1}^{h-1}\left[\frac{f_{\text {step }}^{*}\left(\mathcal{X}_{m}^{(k)}\right)}{2^{h-k-1}}\right], \forall h>1 \\
& \mathcal{X}_{m}^{(1)}=x_{m}^{(1)}, \\
& \mathcal{U}_{h}=u_{h}+\sum_{k=1}^{h-1}\left[\frac{u_{k}}{2^{h-k}}-\frac{f_{\text {step }}^{*}\left(\mathcal{X}_{\mathrm{m}}^{(k)}\right)}{2^{h-k-1}}\right], \forall h>1 \\
& \mathcal{U}_{1}=u_{1} .
\end{aligned}
$$

\section{APPENDIX B: SINGLE-ROUND ERROR ESTIMATION}

Here we present further details for a single round of syndrome extraction (SE) and error estimation.

\section{Wave function after the $q$-SE circuit}

First, we show that the state after the $q$-SE circuit is approximated by simple transformation of its initial wave function.

Theorem 1. A $q$-SE circuit with the measurement result $x_{m}$ approximately transforms an input qubit wave function according to

$$
Q_{\alpha}^{\vec{\Delta}}(x-u) \rightarrow Q_{s\left(x_{m}\right)+\frac{\alpha}{2}}^{\vec{\Delta}^{\prime}}\left(x-\frac{u}{2}\right) .
$$

Proof. The two-mode input to the $q$-SE circuit has the following wave function:

$$
\Phi_{\text {in }}(x, y)=Q_{\alpha}^{\vec{\Delta}}(x-u) \psi_{+}^{\vec{\Delta}}(y),
$$

where the qubit $Q_{\alpha}^{\vec{\Delta}}=a_{0} \psi_{0+\alpha}^{\vec{\Delta}}+a_{1} \psi_{1+\alpha}^{\vec{\Delta}}$ has an error shift $u$ and a relative peak shift $\alpha$.

After the beamsplitter and squeezer of the $q$-SE circuit, the wave function is

$$
\Phi(x, y)=Q_{\alpha}^{\vec{\Delta}}\left(x+\frac{y}{\sqrt{2}}-u\right) \psi_{+}^{\vec{\Delta}}\left(-x+\frac{y}{\sqrt{2}}\right),
$$


which may be expanded as

$$
\begin{aligned}
\Phi(x, y)= & \sum_{\mu, n, m} a_{\mu} N_{\mu} G_{\frac{1}{\kappa}}[(2 n+\mu+\alpha) \sqrt{\pi}] \\
& \times G_{\Delta}\left[x-u+\frac{y}{\sqrt{2}}-(2 n+\mu+\alpha) \sqrt{\pi}\right] \\
& \times G_{\frac{1}{\kappa}}(m \sqrt{\pi}) G_{\Delta}\left(-x+\frac{y}{\sqrt{2}}-m \sqrt{\pi}\right),
\end{aligned}
$$

where the sums are for integers $n, m$, and $\mu \in\{0,1\}$.

We now identify that this may be written in terms of unrotated GKP wave functions. To do so, we regroup Gaussian functions of the same width by completing the square in the exponent, resulting in

$$
\begin{aligned}
\Phi(x, y) \propto & \sum_{\beta} \psi_{-\beta+\frac{\alpha}{2}}^{\vec{\Delta}^{\prime}}\left(\frac{y}{\sqrt{2}}-\frac{u}{2}\right) \\
& \times\left[a_{0} \psi_{\beta+\frac{\alpha}{2}}^{\vec{\Delta}^{\prime}}\left(x-\frac{u}{2}\right)+a_{1} \psi_{1+\beta+\frac{\alpha}{2}}^{\vec{\Delta}^{\prime}}\left(x-\frac{u}{2}\right)\right],
\end{aligned}
$$

where the sum is over $\beta \in\left\{-\frac{1}{2}, 0, \frac{1}{2}, 1\right\}$. This wave function is a superposition of two-mode GKP states with relative peaks shifts differing by $\beta$. Note also that the initial error $u$ is now shared equally by the modes. To simplify the expression, we have used the approximation that the normalization constants are equal: $\frac{1}{N_{0}} \approx \frac{N_{0}}{N_{1}^{2}} \approx \frac{N_{0}}{N_{1 / 2} N_{-1 / 2}}$.

For the measurement result $x_{m}$, the output wave function is proportional to $\Phi\left(x, x_{m}\right)$. For a particular $x_{m}$, one term from Eq. (B4) is much larger than the others, since the weighting functions are positive valued and nearly orthogonal if $\Delta$ is sufficiently smaller than $\sqrt{\pi} / 2$. The dominant term is given by $\beta=s\left(x_{m}-\frac{u}{\sqrt{2}}-\alpha \sqrt{\frac{\pi}{2}}\right)$, and therefore we approximate that the output state as that given by the most likely case $u=0$.

The average fidelity of this approximation is given by the average value of $\left|\psi_{s\left(x_{m}\right)+\frac{\alpha}{2}}^{\vec{\Delta}^{\prime}}\right|^{2}$ taken over the joint probability distribution $\mathbb{P}\left(x_{m}, u\right)=\mathbb{P}\left(x_{m} \mid u\right) \mathbb{P}(u)$ (these distributions are discussed below).

A similar result is obtained for the $p$-quadrature syndrome extraction. In this case, however, the widths undergo the inverse transformation according to $(\Delta, \kappa) \rightarrow(\Delta \sqrt{2}, \kappa / \sqrt{2})$. Appropriately designed $q$-SE and $p$-SE steps can therefore be used sequentially to prevent any overall change in the widths. In particular, we begin with a $q$-SE step using a qubit and an auxiliary state of width $(\Delta, \kappa)$. The following $p$-SE step then uses an auxiliary state of width $(\Delta / \sqrt{2}, \kappa / \sqrt{2})$, and the final state from this has the same width as the initial one. We note that the effect of width reduction through syndrome extraction cannot be observed in the $\Delta=0$ unphysical state's calculation.

\section{Wave function after error, $q-\mathrm{SE}$ and $p-\mathrm{SE}$}

We now extend the above calculation to include a subsequent $p$-SE step. In particular, we show the transformation of the input state is well approximated by

$$
Q^{\vec{\Delta}}(x-u) e^{i v x} \rightarrow Q^{\vec{\Delta}}\left[x-\theta\left(x_{\mathrm{m}}, u\right)\right] e^{i \theta\left(p_{\mathrm{m}}, v\right) x},
$$

where the shift function $\theta$ is defined as

$$
\theta(y, b)=b / 2-f_{\text {step }}^{*}(y) .
$$

The two-mode state after the $p$-SE beamsplitter and squeezer is given by

$$
\begin{aligned}
\Phi(x, z)= & \mathrm{e}^{i\left(\frac{x}{2}+\frac{z}{\sqrt{2}}\right) v} \psi_{0}^{\vec{\Delta}^{\prime}}\left(\frac{x}{2}-\frac{z}{\sqrt{2}}\right) \\
& \times Q^{\vec{\Delta}^{\prime}}\left(\frac{x}{2}+\frac{z}{\sqrt{2}}-\frac{u}{2}+f_{\text {step }}^{*}\left(x_{\mathrm{m}}-u / \sqrt{2}\right)\right),
\end{aligned}
$$

where the quadrature $z$ corresponds to the mode to be measured. This $p$-quadrature measurement is achieved using the Fourier transform of the two-mode wave function:

$$
\begin{aligned}
& \mathcal{F}\{\Phi(x, z)\}=\mathcal{F}_{y \rightarrow p-\frac{p_{\mathrm{m}}}{\sqrt{2}}}\left\{\psi_{0}^{\vec{\Delta}^{\prime}}(y)\right\} \\
& \quad \times \mathcal{F}_{w \rightarrow p+\frac{p_{\mathrm{m}}}{\sqrt{2}}}\left\{\mathrm{e}^{i w v} Q^{\vec{\Delta}^{\prime}}\left(w-\frac{u}{2}+f_{\text {step }}^{*}\left(x_{\mathrm{m}}-u / \sqrt{2}\right)\right)\right\} .
\end{aligned}
$$

where the transform is taken for variables $(x, z) \rightarrow\left(p, p_{m}\right)$, and on the right-hand side we use the change of variables $w=$ $\frac{x}{2}+\frac{z}{\sqrt{2}}$ and $y=\frac{x}{2}-\frac{z}{\sqrt{2}}$. The shift property of the transform yields

$$
\begin{aligned}
\tilde{\Phi}\left(p, p_{m}\right)= & \tilde{\psi}_{0}^{\vec{\Delta}^{\prime}}\left(p-\frac{p_{m}}{\sqrt{2}}\right) \tilde{Q}_{0}^{\vec{\Delta}^{\prime}}\left(p+\frac{p_{m}}{\sqrt{2}}-v\right) \\
& \times e^{-i p\left[u / 2-f_{\text {step }}^{*}\left(x_{m}-u / \sqrt{2}\right)\right]},
\end{aligned}
$$

where we denote Fourier transforms as $\tilde{g}=\mathcal{F}\{g\}$.

Since the finite-energy GKP states are approximate eigenstates of the Fourier transform, the first two terms are similar to the product of two rotated GKP states, treated above. Using similar methods, we find the approximate relationship

$$
\begin{aligned}
\tilde{\psi}_{0}^{\vec{\Delta}^{\prime}}\left(p-\frac{p_{m}}{\sqrt{2}}\right) \tilde{Q}^{\vec{\Delta}^{\prime}}\left(p+\frac{p_{m}}{\sqrt{2}}-v\right) \\
\propto \tilde{Q}^{\vec{\Delta}}\left[p-\frac{v}{2}+f_{\text {step }}^{*}\left(p_{m}-v / \sqrt{2}\right)\right] .
\end{aligned}
$$

We now simplify the step function by noting that $f_{\text {step }}^{*}\left(p_{m}-\right.$ $v / \sqrt{2}) \approx f_{\text {step }}^{*}\left(p_{m}\right)$. The success probability of this approximation is discussed later. Finally, we take the inverse Fourier transform to find the desired result given by Eq. (B5).

\section{Bayesian estimation of the $q$-quadrature error}

In order to estimate the unknown shift error, we wish to determine its posterior probability distribution. To do so, we use the prior probability distribution, specified by the error model, and a likelihood function, $\mathbb{P}\left(x_{m} \mid u\right)$, that we now calculate from the wave function. 
Theorem 2. The probability of measurement result $x_{m}$ from a $q$-SE circuit with the initial state $\psi_{\alpha}^{\vec{\Delta}}(x-u)$ is

$$
\mathbb{P}\left(x_{m} \mid u\right) \propto \psi_{+}^{\vec{\Delta}}\left(\sqrt{2} x_{m}-u\right) .
$$

Proof. We start with the two-mode wave function given in Eq. (B4). The probability for measurement result $x_{m}$ is

$$
\begin{aligned}
\mathbb{P}\left(x_{m} \mid u\right) & =\int_{x \in \mathbb{R}}\left|\Phi\left(x, x_{m}\right)\right|^{2} d x \\
& \approx \sum_{\beta}\left|\psi_{\beta}^{\vec{\Delta}^{\prime}}\left(\frac{x_{m}}{\sqrt{2}}-\frac{u}{2}\right)\right|^{2},
\end{aligned}
$$

where in the second step we neglect overlap of the GKP wave functions to approximate $\left|\sum \psi_{\beta}\right|^{2} \approx \sum\left|\psi_{\beta}\right|^{2}$.

An alternate expression is obtained from the relationships

$$
\begin{gathered}
\sum_{\beta \in\{0,1\}}\left|\psi_{\beta}^{\vec{\Delta}^{\prime}}\left(\frac{x_{m}}{\sqrt{2}}-\frac{u}{2}\right)\right|^{2} \propto \psi_{0}^{\vec{\Delta}}(0) \psi_{0}^{\vec{\Delta}}\left(\sqrt{2} x_{m}-u\right), \\
\sum_{\beta \in\left\{ \pm \frac{1}{2}\right\}}\left|\psi_{\beta}^{\vec{\Delta}^{\prime}}\left(\frac{x_{m}}{\sqrt{2}}-\frac{u}{2}\right)\right|^{2} \propto \psi_{0}^{\vec{\Delta}}(0) \psi_{1}^{\vec{\Delta}}\left(\sqrt{2} x_{m}-u\right),
\end{gathered}
$$

which may be shown by reindexing akin to the derivation of Eq. (B10). The addition of these two equations yields the desired result. tion is

Using Bayes' theorem, the posterior probability distribu-

$$
\begin{aligned}
\mathbb{P}\left(u \mid x_{m}\right) & \propto \mathbb{P}\left(x_{m} \mid u\right) \mathbb{P}(u) \\
& \propto \psi_{+}^{\vec{\Delta}}\left(\sqrt{2} x_{m}-u\right) e^{-\frac{u^{2}}{2 \sigma_{0}^{2}}},
\end{aligned}
$$

where in the last step we have explicitly written the Gaussian error model. The unknown shift can now be estimated from Eq. (B13) using an appropriate estimator, such as the mean of $\mathbb{P}\left(u \mid x_{m}\right)$. For a given $x_{m}$, along with $\Delta \ll \sqrt{\pi}$ and $u \ll 1$, $\mathbb{P}\left(u \mid x_{m}\right)$ can be well approximated by a normal distribution:

$$
\mathbb{P}\left(u \mid x_{m}\right) \approx \mathcal{N}\left(\frac{\sqrt{2} \sigma_{0}^{2} x_{m}-\sqrt{\pi} \sigma_{0}^{2}\left\lfloor\frac{\sqrt{2} x_{m}}{\sqrt{\pi}}\right\rceil}{\Delta^{2}+\sigma_{0}^{2}}, \frac{\Delta^{2} \sigma_{0}^{2}}{\Delta^{2}+\sigma_{0}^{2}}\right) .
$$

The approximation of the posterior as a normal distribution given reasonable parameter choices is an important step used below in multiround calculations.

\section{Bayesian estimation of $q$ - and $p$-quadrature errors}

We now expand on the previous section to estimate an unknown shift in both quadratures.

Theorem 3. The probability of measurement results $x_{m}$ and $p_{m}$ from sequential $q$-SE and $p$-SE circuits with the initial state $e^{i v x} \psi_{\alpha}^{\vec{\Delta}}(x-u)$ is

$$
\mathbb{P}\left(x_{m}, p_{m} \mid u, v\right) \propto \psi_{+}^{\vec{\Delta}}\left(\sqrt{2} x_{m}-u\right) \psi_{+}^{(2 \Delta, \kappa / 2)}\left(\sqrt{2} p_{m}-v\right) .
$$

Proof. Starting from the wave function in Eq. (B4), the probability of $p_{m}$ is

$$
\begin{aligned}
\mathbb{P}\left(p_{m} \mid u, v, x_{m}\right) & =\int_{p \in \mathbb{R}}\left|\tilde{\Phi}\left(p, p_{m}\right)\right|^{2} d p \\
& \propto \psi_{+}^{(2 \Delta, \kappa / 2)}\left(\sqrt{2} p_{m}-v\right),
\end{aligned}
$$

where the second step follows by analogy to Eqs. (B2)(B4) and (B12)-(B15). The desired result follows from the relationship of conditional probabilities $\mathbb{P}\left(x_{m}, p_{m} \mid u, v\right)=$ $\mathbb{P}\left(x_{m} \mid u, v\right) \mathbb{P}\left(p_{m} \mid u, v, x_{m}\right)$.

Note that $\mathbb{P}\left(p_{m} \mid u, v, x_{m}\right)$ is independent of $x_{m}$ and $u$. We can therefore write $\mathbb{P}\left(x_{m}, p_{m} \mid u, v\right)=\mathbb{P}\left(x_{m} \mid u\right) \mathbb{P}\left(p_{m} \mid v\right)$, where the $\mathbb{P}\left(p_{m} \mid v\right)$ differs from $\mathbb{P}\left(x_{m} \mid u\right)$ according to the transformations $\Delta \rightarrow 2 \Delta$ and $\kappa \rightarrow \kappa / 2$.

\section{APPENDIX C: MULTIROUND ERROR ESTIMATION}

We now consider $M$ rounds of error followed by $q$-SE and $p$-SE circuits, with an aim to estimate the shift error on the final state.

\section{Wave function after multiple rounds}

As shown for the single-round scenario, the wave function after each round is a shifted version of the input. An expression for the shift after each round is calculated iteratively. After round $h$, with a history of measurements $\vec{x}_{\mathrm{m}}=$ $\left(x_{m}^{(1)}, \ldots, x_{m}^{(h)}\right)$ and shift errors $\vec{u}=\left(u_{1}, \ldots, u_{h}\right)$, the total $q$ shift is

$$
\theta_{h}\left(\vec{x}_{m}, \vec{u}\right)=\sum_{k=1}^{h} \frac{1}{2^{h-k}} \theta\left[\mathcal{X}_{k}\left(\vec{x}_{m}\right), u_{k}\right],
$$

where $\theta$ is defined in Eq. (B6) and

$$
\mathcal{X}_{m}^{(k)}\left(\vec{x}_{m}\right)=x_{m}^{(k)}+\frac{1}{\sqrt{2}} \sum_{j=1}^{k-1}\left[\frac{f_{\text {step }}^{*}\left[\mathcal{X}_{m}^{(j)}\left(\vec{x}_{m}\right)\right]}{2^{k-j-1}}\right]
$$

for $h>1$, and $\mathcal{X}_{m}^{(1)}=x_{m}^{(1)}$. The parameter $\mathcal{X}_{m}^{(k)}$ can be interpreted as the measurement outcome $x_{m}^{(k)}$ transformed to account for previous measurement-induced shifts.

Equation (C1) can also be expanded as

$$
\begin{aligned}
\theta_{h}\left(\vec{x}_{m}, \vec{u}\right) & =\sum_{j=1}^{h}\left[\frac{u_{k}}{2^{h-j+1}}\right]-\sum_{k=1}^{h}\left[\frac{f_{\text {step }}^{*}\left[\mathcal{X}_{m}^{(k)}\left(\vec{x}_{m}\right)\right]}{2^{h-k}}\right] \\
& =\theta_{h}^{\operatorname{err}}(\vec{u})-\theta_{h}^{\text {step }}\left(\vec{x}_{m}\right),
\end{aligned}
$$

which identifies the total shift as composed of a known measurement-induced shift $\theta_{h}^{\text {step }}\left(\vec{x}_{m}\right)$ and the accumulation of unknown error $\theta_{h}^{\text {err }}(\vec{u})$. The aim of our error estimation after $M$ rounds is to use $\vec{x}_{m}$ to estimate $\theta_{M}^{\operatorname{err}}(\vec{u})$.

An analogous approach to the $p$ shifts leads to the wave function

$$
e^{i \theta_{h}\left(\vec{p}_{m}, \vec{v}\right) x} Q^{\vec{\Delta}}\left[x-\theta_{h}\left(\vec{x}_{m}, \vec{u}\right)\right]
$$

after $h$ rounds, for an initial qubit state $Q^{\vec{\Delta}}(x)$. This is assuming that $f_{\text {step }}$ is independent of $u_{k}$, which is true if $f_{\text {step }}^{*}\left(x_{m}\right)=$ $f_{\text {step }}^{*}\left(x_{m}-u / \sqrt{2}\right)$. We discuss the error of such an approximation in a later section. 


\section{Total qubit drift}

Without active corrective shift at each round of QEC, the magnitude of the total drift of the state in phase space can be bounded. Using the fact that $\left|f_{\text {step }}^{*}\right| \leqslant \sqrt{\pi}$ and taking the variance of each the error shifts to be $\sigma_{0}^{2}$, an application of the triangle inequality gives the following:

$$
\begin{aligned}
& \left|\theta\left(x_{m}^{(h)}, \mathcal{U}_{h}\right)\right|,\left|\theta\left(p_{m}^{(h)}, \mathcal{V}_{h}\right)\right| \\
& \quad \leqslant 2 \sqrt{\pi}\left(1-2^{-h}\right)+\left|\mathcal{N}\left(0, \sigma_{0}^{2} \frac{\left(1-4^{-h}\right)}{3}\right)\right|,
\end{aligned}
$$

where $\mathcal{N}(\mu, V)$ is a Gaussian random variable with mean $\mu$ and variance $V$. The quantities $1-4^{-h}$ and $1-2^{-h}$ converge very quickly to 1 as $h$ increases, meaning that for multiple rounds we have

$$
\left|\theta\left(x_{m}^{(h)}, \mathcal{U}_{h}\right)\right|,\left|\theta\left(p_{m}^{(h)}, \mathcal{V}_{h}\right)\right| \leqslant 2 \sqrt{\pi}+\left|\mathcal{N}\left(0, \frac{\sigma_{0}^{2}}{3}\right)\right| .
$$

This means that even without active error-corrective shift after each round of syndrome extraction, the GKP qubit is expected to drift by a maximum of $2 \sqrt{\pi}$ in phase space along with some Gaussian random variable with variance $\sigma_{0}^{2} / 3$ in the worstcase scenario. Consequently, the energy of the physical system is not divergent if we extend the QEC for many rounds, if errors are sufficiently small at each round.

\section{Memory-assisted decoder}

The probability distributions for the $x$ and $p$ measurement values obtained in the $h$ th round, given all the previous measurement results and displacement shifts, are given by expressions similar in spirit to that in Theorem 2:

$$
\begin{aligned}
& \mathbb{P}\left(x_{m}^{(h)} \mid u_{1}, \ldots, u_{h}, x_{m}^{(1)}, \ldots, x_{m}^{(h-1)}\right) \propto \psi_{+}^{(\Delta, \Delta)}\left(\sqrt{2} x_{m}^{(h)}-\mathcal{U}_{h}\right), \\
& \mathbb{P}\left(p_{m}^{(h)} \mid v_{1}, \ldots, v_{h}, p_{m}^{(1)}, \ldots, p_{m}^{(h-1)}\right) \propto \psi_{+}^{(2 \Delta, \Delta / 2)}\left(\sqrt{2} p_{m}^{(h)}-\mathcal{V}_{h}\right) .
\end{aligned}
$$

Using Bayes' theorem to flip these distributions, for $M$ rounds we obtain

$$
\begin{aligned}
\mathbb{P}_{M}\left(\vec{u}, \vec{v} \mid \vec{x}_{m}, \vec{p}_{m}\right) & =\mathbb{P}_{M}^{(q)}\left(\vec{u} \mid \vec{x}_{m}\right) \mathbb{P}_{M}^{(p)}\left(\vec{v} \mid \vec{p}_{m}\right), \\
\mathbb{P}_{M}^{(q)}\left(\vec{u} \mid \vec{x}_{m}\right) & \propto \prod_{h=1}^{M} \psi_{+}^{\vec{\Delta}}\left(\sqrt{2} x_{m}^{(h)}-\mathcal{U}_{h}\right) G_{\sigma_{0}}\left(u_{h}\right), \\
\mathbb{P}_{M}^{(p)}\left(\vec{v} \mid \vec{p}_{m}\right) & \propto \prod_{h=1}^{M} \psi_{+}^{(2 \Delta, \Delta / 2)}\left(\sqrt{2} p_{m}^{(h)}-\mathcal{V}_{h}\right) G_{\sigma_{0}}\left(v_{h}\right) .
\end{aligned}
$$

We can see that the $q$ - and $p$-quadrature equations have the similar forms and can be unchangeable $(q \leftrightarrow p)$ if $u \leftrightarrow$ $v, x_{m} \leftrightarrow p_{m}$, and $(\Delta, \kappa) \leftrightarrow(2 \Delta, \kappa / 2)$, so we focus on the $q$-quadature version for now. The PDF for $\vec{u}$ is well approximated by a multivariate Gaussian distribution, a step known as Laplace's approximation in statistics, which we write as

$$
\mathbb{P}_{M}^{(q)}\left(\vec{u} \mid \vec{x}_{m}\right) \approx \mathcal{N}(\overrightarrow{\tilde{u}}, \Sigma)
$$

for mean vector $\overrightarrow{\tilde{u}}$ and covariance matrix $\Sigma$.

We derive the inverse of the covariance matrix, $\Sigma^{-1}$, exactly by completing the square of the exponents of the
Gaussians in Eq. (C9):

$$
\begin{aligned}
& \left(\Sigma^{-1}\right)_{\alpha, \beta}=\left(\frac{\delta_{\alpha, \beta}}{\sigma_{0}^{2}}+\frac{1}{\Delta^{2}} \sum_{h=m_{\alpha, \beta}}^{M} \frac{1}{4^{h} 2^{-(\alpha+\beta)}}\right), \\
& m_{\alpha, \beta}=\max \{\alpha, \beta\} .
\end{aligned}
$$

To derive $\overrightarrow{\tilde{u}}$ via completing the square, we need to invert the matrix $\Sigma^{-1}$. It is generally hard to invert an $M \times M$ symmetric matrix for an arbitrary $M$, but we can approximate the covariance matrix via a Neumann series expansion of the matrices, under the assumption of small displacements. We find the covariance matrix has the following components:

$$
\Sigma_{\alpha, \beta} \approx \sigma_{0}^{2} \delta_{\alpha, \beta}-\frac{\sigma_{0}^{4}}{\Delta^{2}} \sum_{h=m_{\alpha, \beta}}^{M} \frac{1}{4^{h} 2^{-(\alpha+\beta)}}+O\left(\frac{\sigma_{0}^{6}}{\Delta^{4}}\right),
$$

and we note that this Neumann series converges if $\frac{\sigma_{0}}{\Delta}<\frac{1}{2}$ (which works for all $M$ ). By ignoring from order $O\left(\frac{\sigma_{0}^{6}}{\Delta^{6}}\right)$ upwards in the expansion, we compute

$$
\begin{aligned}
\tilde{u}_{k}= & \left(\frac{\sigma_{0}}{\Delta}\right)^{2}\left\{2^{k} \sum_{j=1}^{M} \frac{F_{j}}{2^{j}}-\left(\frac{\sigma_{0}}{\Delta}\right)^{2} \sum_{h=1}^{M}\right. \\
& \left.\times\left[\left(\sum_{n=m_{k, h}}^{M} \frac{2^{k+h}}{4^{n}}\right)\left(\sum_{j=h}^{M} \frac{F_{j}}{2^{j}}\right)\right] 2^{h}\right\},
\end{aligned}
$$

where $\quad m_{k, h}=\max \{k, h\} \quad$ and $\quad F_{h}=\sqrt{2} \mathcal{X}_{m}^{(h)}-$ $\sqrt{\pi}\left\lfloor\sqrt{2} \mathcal{X}_{m}^{(h)} / \sqrt{\pi}\right\rceil$, which is Eq. (14) in the main text.

Now that we have characterized the multivariate Gaussian noise model (multivariate due to many rounds) by its covariance matrix, we wish to estimate the random part of the total displacement after $M$ rounds, given by $\sum_{k=1}^{M} u_{k} / 2^{M-k+1}$. The corresponding PDF is

$$
\mathbb{P}\left(\sum_{k=1}^{M} \frac{u_{k}}{2^{M-k+1}} \mid \vec{x}_{m}\right)=\mathcal{N}\left(\vec{a} \cdot \overrightarrow{\tilde{u}}, \vec{a}^{\mathrm{T}} \cdot \Sigma \cdot \vec{a}\right),
$$

with $a_{k}=2^{-(M+1-k)}$.

We use the mean of this posterior distribution as the MMSE estimator, with uncertainty given by the variance

$$
V_{q}=\operatorname{Var}\left(\sum_{k=1}^{M} \frac{u_{k}}{2^{M-k+1}}\right)=\vec{a}^{\mathrm{T}} \cdot \Sigma \cdot \vec{a} .
$$

Neglecting terms of order $\left(\frac{\sigma_{0}^{6}}{\Delta^{4}}\right)$ and higher, we compute

$$
\begin{aligned}
V_{q}(M)= & \frac{\sigma_{0}^{2}}{3}\left[\left(1-4^{-M}\right)+\left(\frac{2 \sigma_{0}}{3 \Delta}\right)^{2}\right. \\
& \left.\times\left[4^{-2 M}+3(1+2 M) 4^{-M}-4\right]\right] .
\end{aligned}
$$

We can see that $V_{q} \rightarrow \frac{\sigma_{0}^{2}}{3}$, very quickly as $M$ grows. Note that using measurement results from all rounds of syndrome measurements allows the error to be estimated more precisely than is achievable otherwise. 


\section{APPENDIX D: APPROXIMATIONS AND QUBIT FIDELITY}

In our calculations, two significant approximations are made in expressing the qubit wave function emerging from a syndrome extraction circuit as a displacement of the incoming wave function. For the single-round case, these are discussed following Eq. (B4). First, in identifying the dominant GKPlike term in the resulting wave function, we approximate that $f_{\text {step }}^{*}\left(x_{m}-u / \sqrt{2}\right)=f_{\text {step }}^{*}\left(x_{m}\right)$. Second, we keep only the dominant term in the sum of Eq. (B4). Each of these approximations reduces the fidelity of our description of the final state.

\section{Tracking error}

Unlikely measurement outcomes and unusually large displacement errors can cause an incorrect identification of the dominant GKP-like term, which we refer to as a tracking error. In this section, we estimate the probability that our description of the state after one or multiple rounds of syndrome extraction, as given in Eq. (C4), does not contain a tracking error.

\section{a. Single round}

For a single round, the joint probability density function of $x_{m}$ and $u$ is

$\mathbb{P}\left(u, x_{m}\right)=C \sum_{n \in \mathbb{Z}} G_{1 / \Delta}(n \sqrt{\pi}) G_{\Delta}\left(\sqrt{2} x_{m}-u-n \sqrt{\pi}\right) G_{\sigma_{0}}(u)$.

No tracking error occurs if $x_{m}$ and $u$ are in the region $\mathcal{S}$ for which $\left|f_{\text {step }}^{*}\left(x_{m}\right)-f_{\text {step }}^{*}\left(x_{m}-u / \sqrt{2}\right)\right|=0$.

We therefore define the success probability for one round and error width $\sigma_{0}$ to be

$$
P_{\text {track }}^{\text {succ }}\left(1, \sigma_{0}\right)=\int_{S} \mathbb{P}\left(u, x_{m}\right) d \mathcal{S},
$$

for which the integration region could be rewritten as $x_{m} \in \mathbb{R}$ and $u \in \mathcal{T}$, where $\mathcal{T}=[-\sqrt{\pi}, 0]+$ $\sqrt{2} \bmod \left(x-\sqrt{\frac{\pi}{2}} / 2, \sqrt{\frac{\pi}{2}}\right)$. The normalization constant $C=\left[\sqrt{2} \pi \sigma_{0} \Delta \Theta_{3}\left(0, e^{-\pi \Delta^{2} / 2}\right)\right]^{-1}, \quad$ where $\Theta_{3} \quad$ is the Jacobi-Theta function of the third kind.

For our example in the main text where $\left(\Delta, \sigma_{0}^{2}\right)=$ $(0.2182,0.0005)$, we calculate $1-P_{\text {track }}^{\text {succ }}(1, \sigma) \approx 1 \times 10^{-5}$.

\section{b. Multiple rounds}

For multiple rounds, the joint probability density function of $\vec{x}_{m}$ and $\vec{u}$ is

$$
\mathbb{P}\left(\vec{u}, \vec{x}_{m}\right)=\prod_{h=1}^{M} \psi_{+}^{\vec{\Delta}}\left(\sqrt{2} x_{m}^{(h)}-\mathcal{U}_{h}\right) G_{\sigma_{0}}\left(u_{h}\right) .
$$

Similar to the single-round case, the probability for $M$ successful rounds is given by

$$
P_{\text {track }}^{\text {succ }}\left(M, \sigma_{0}\right)=\int_{\mathcal{L}} \mathbb{P}\left(\vec{u}, \vec{x}_{m}\right) d \mathcal{L},
$$

where $\mathcal{L}$ is the region defined by $f_{\text {step }}^{*}\left(\mathcal{X}_{\mathrm{m}}^{(j)}\right)=f_{\text {step }}^{*}\left(x_{m}^{(j)}-\right.$ $\left.\mathcal{U}_{j} / \sqrt{2}\right), \forall 1<j<M$.

This calculation is simplified by changing variables to $\overrightarrow{\mathcal{X}}$ and $\vec{U}$, where $\mathbb{U}_{h}=\sum_{k=1} \frac{u_{k}}{2^{h-k}}$ for $h>1$ and $\mathbb{U}_{1}=u_{1}$, which leads to

$$
\begin{gathered}
P_{\text {track }}^{\text {succ }}\left(M, \sigma_{0}\right)>\int_{\mathcal{L}} \prod_{h=2}^{M}\left[\psi_{+}^{\vec{\Delta}}\left(\sqrt{2} \mathcal{X}_{m}^{(h)}-\mathbb{U}_{h}\right) G_{\frac{2 \sigma_{0}}{\sqrt{5}}}\left(\mathbb{U}_{h}\right)\right] \\
\times \psi_{+}^{\vec{\Delta}}\left(\sqrt{2} \mathcal{X}_{m}^{(1)}-\mathbb{U}_{1}\right) G_{\sigma_{0}}\left(\mathbb{U}_{h}\right) d^{M} \overrightarrow{\mathcal{X}} d^{M} \overrightarrow{\mathbb{U}}
\end{gathered}
$$

Note the multidimensional integral is now separated into a product of single integrals over the integration regions $f_{\text {step }}^{*}\left(\mathcal{X}_{\mathrm{m}}^{(j)}\right)=f_{\text {step }}^{*}\left(\mathcal{X}_{\mathrm{m}}^{(j)}-\mathbb{U}_{j} / \sqrt{2}\right)$ for all $2<j<M$. A convenient bound lower bound can be written in terms of single-round probabilities:

$$
P_{\text {track }}^{\text {succ }}\left(M, \sigma_{0}\right)>\left[P_{\text {track }}^{\text {succ }}\left(1, \frac{2 \sigma_{0}}{\sqrt{5}}\right)\right]^{M} .
$$

For $\left(\Delta, \sigma_{0}^{2}\right)=(0.2182,0.0005)$ and $M=200$, we calculate $1-P_{\text {track }}^{\text {succ }}\left(M, \sigma_{0}\right) \approx 3 \times 10^{-3}$.

\section{Truncation error}

The approximation that only the dominant term in the state survives at each round of syndrome extraction leads to a probability that our decoder fails. We use an approximation of this probability to bound the long term fidelity of a state recovered using information from the memory-assisted decoder. In particular, we approximate the state after each round of syndrome extraction by decohering the dominant term with respect to the rest of the state: $p \hat{\rho}_{\text {dom. }}+(1-p) \hat{\rho}_{\text {junk }}$, where $p$ is the phenomenological success probability per round, $\hat{\rho}_{\text {dom }}$. is the dominant term we keep and $\hat{\rho}_{\text {junk }}$ is the state we throw away in the truncation.

\section{a. Single round}

The wave function emerging from a single syndromeextraction circuit, described by Eq. (B4), can be expressed in ket notation as

$$
\left|\Phi\left(x \mid u, x_{m}\right)\right\rangle=R\left(u, x_{m}\right) \sum_{\gamma} \psi_{\gamma}^{\vec{\Delta}^{\prime}}\left(\frac{x_{m}}{\sqrt{2}}-\frac{u}{2}\right)\left|Q_{\gamma}^{\vec{\Delta}}\right\rangle,
$$

where $R\left(u, x_{m}\right)$ is a normalization constant. We note the dominant term as $\left|Q_{\beta}^{\vec{\Delta}}\right\rangle$ and calculate its amplitude as

$$
A(\beta)=R\left(u, x_{m}\right) \sum_{\gamma} \psi_{\gamma}^{\vec{\Delta}^{\prime}}\left(\frac{x_{m}}{\sqrt{2}}-\frac{u}{2}\right)\left\langle Q_{\beta}^{\vec{\Delta}} \mid Q_{\gamma}^{\vec{\Delta}}\right\rangle .
$$
by

The average success of the truncated description is given

$$
P_{\text {trunc }}^{\text {succ }}\left(1, \sigma_{0}\right)=\int_{\text {all space }}|A(\beta)|^{2} \mathbb{P}\left(u, x_{m}\right) d u d x_{m} .
$$

For the example $\left(\Delta, \sigma_{0}^{2}\right)=(0.2182,0.0005)$, we calculate $1-P_{\text {trunc }}^{\text {succ }}\left(1, \sigma_{0}\right) \approx 5 \times 10^{-5}$. 
Algorithm 1. Estimation of the $q$-quadrature error

\begin{tabular}{l} 
Initialize empty vectors $\overrightarrow{\mathcal{X}}_{\mathrm{m}}, \vec{F}, \overrightarrow{\tilde{u}}$ \\
for $h=1$ to $M$ do \\
$\quad$ Receive data $x_{m}^{(h)}$ \\
Compute $\mathcal{X}_{m}^{(h)}=x_{m}^{(h)}+\frac{1}{\sqrt{2}} \sum_{k=1}^{h-1} \frac{f_{\text {step }}^{*}\left(\mathcal{X}_{m}^{(k)}\right)}{2^{h-k-1}}$ \\
Compute $F_{h}=\sqrt{2} \mathcal{X}_{m}^{(h)}-\sqrt{\pi}\left[\frac{\sqrt{2} \mathcal{X}_{t} m^{(h)}}{\sqrt{\pi}}\right\rceil$ \\
Append $\mathcal{X}_{m}^{(h)}$ to $\overrightarrow{\mathcal{X}}_{m}$ and $F_{h}$ to $\vec{F}$ \\
end for \\
Compute total displacement $\sum_{k=1}^{M} \frac{\tilde{u}_{k}}{2^{M-k+1}}$ where \\
$\tilde{u}_{k}=\left(\frac{\sigma_{0}}{\Delta}\right)^{2}\left\{2^{k} \sum_{j=1}^{M} \frac{F_{j}}{2^{j}}-\left(\frac{\sigma_{0}}{\Delta}\right)^{2} \sum_{h=1}^{M}\left[\left(\sum_{n=m_{k, h}}^{M} \frac{2^{k+h}}{4^{n}}\right)\left(\sum_{j=h}^{M} \frac{F_{j}}{2^{j}}\right)\right] 2^{h}\right\}$ \\
\hline
\end{tabular}

\section{b. Multiple rounds}

Similarly, we estimate a success probability after $M$ rounds as

$$
\begin{aligned}
P_{\text {trunc }}^{\text {succ }}\left(M, \sigma_{0}\right)= & \prod_{h=1}^{M} \int \sum_{\beta_{h}}\left|A\left(\beta_{h}\right)\right|^{2} G_{\sigma_{0}}\left(u_{h}\right) \\
& \times \psi_{+}^{\vec{\Delta}}\left(\sqrt{2} x_{m}^{(h)}-\mathcal{U}_{h}\right) d \vec{u} d \vec{x}_{m} .
\end{aligned}
$$

As in Eq. (D6), we bound the multiround probability in terms of the single-round expression

$$
P_{\text {trunc }}^{\text {succ }}\left(M, \sigma_{0}\right)>\left[P_{\text {trunc }}^{\text {succ }}\left(1,2 \sigma_{0} / \sqrt{5}\right)\right]^{M} .
$$

For $\left(\Delta, \sigma_{0}^{2}\right)=(0.2182,0.0005)$ and $M=200$, we calculate $1-P_{\text {trunc }}^{\text {succ }}\left(M, \sigma_{0}\right) \approx 1 \times 10^{-2}$.

\section{Total error and state fidelity}

To estimate a final qubit fidelity, as in the example shown in Fig. 3, we first calculate the fidelity $F_{\rho}$ from the output state described by our Bayesian estimation procedure, following the approach of Pantaleoni et al. [17]. We then assume that a tracking, truncation, or syndrome error results in a complete depolarized qubit with probability upper-bounded by $1-P_{\text {total }}^{\text {suc }}$. A lower bound on the final qubit fidelity is therefore given by $F=\left(F_{\rho}-\frac{1}{2}\right) P^{\text {succ }}+\frac{1}{2}$.

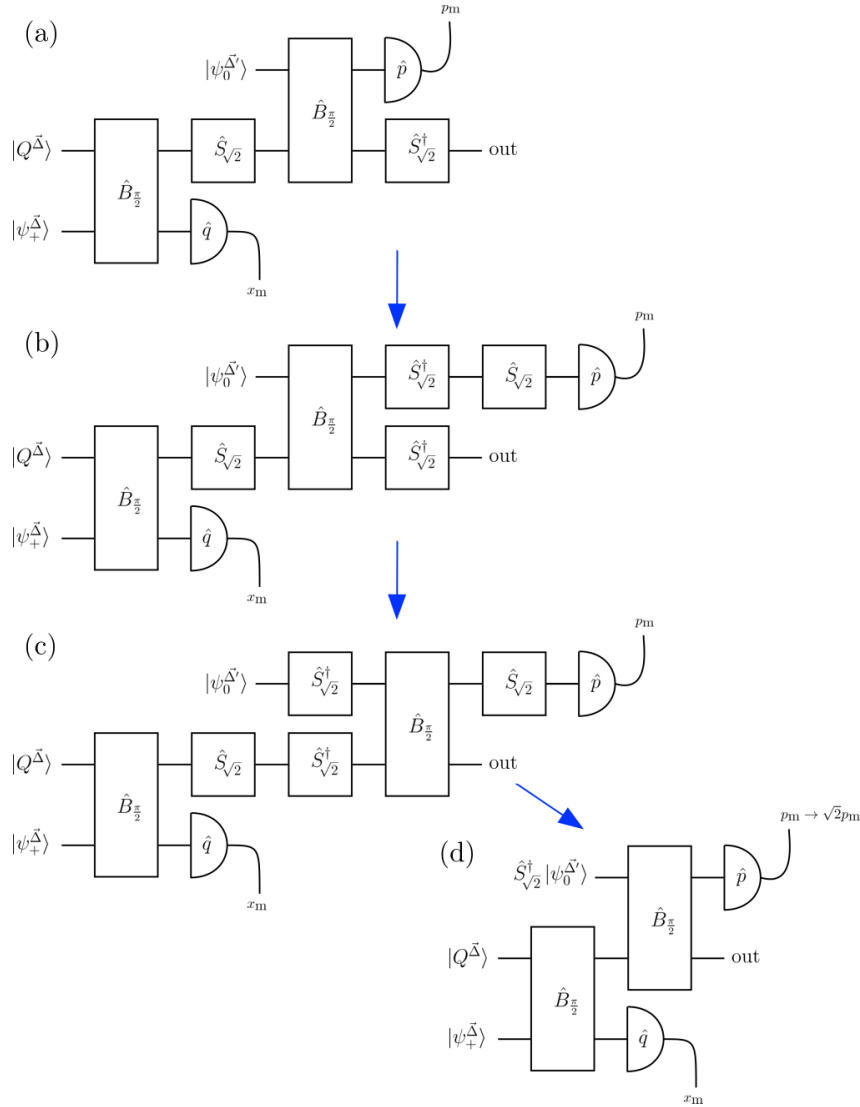

FIG. 4. Diagrammatic derivation of the offline squeezing circuit. In panel (a), we start with the Glancy-Knill syndrome-extraction circuit with the corrective displacement removed. In panel (b), we insert an identity operator before the momentum measurement. In panel (c), we rearrange the 50:50 beamsplitter and squeezers in both modes. Finally in panel (d), we achieve a simplified circuit in which all squeezing is effectively moved to the momentum resource state and interpretation of the momentum measurement result.

\section{APPENDIX E: PSEUDOCODE FOR ERROR ESTIMATION}

After $M$ rounds of syndrome extraction, as depicted in Fig. 2(c), our results can be used to estimate the random contribution of the total displacement error from measurement results $\vec{x}_{m}$ and $\vec{p}_{m}$. Algorithm I gives an explicit description of this calculation for the $q$ quadrature.

\section{APPENDIX F: OFFLINE SQUEEZING DERIVATION}

A diagrammatic derivation of the offline squeezing circuit is shown in Fig. 4. Starting with the Glancy-Knill syndromeextraction circuits, we recompile and reinterpret measurement results to obtain a circuit in which the qubit state interacts only with 50:50 beamsplitters.
[1] S. Lloyd and S. L. Braunstein, Quantum Computation over Continuous Variables, Phys. Rev. Lett. 82, 1784 (1999).
[2] S. L. Braunstein and P. van Loock, Quantum information with continuous variables, Rev. Mod. Phys. 77, 513 (2005). 
[3] C. Weedbrook, S. Pirandola, R. García-Patrón, N. J. Cerf, T. C. Ralph, J. H. Shapiro, and S. Lloyd, Gaussian quantum information, Rev. Mod. Phys. 84, 621 (2012).

[4] J.-i. Yoshikawa, S. Yokoyama, T. Kaji, C. Sornphiphatphong, Y. Shiozawa, K. Makino, and A. Furusawa, Invited Article: Generation of one-million-mode continuous-variable cluster state by unlimited time-domain multiplexing, APL Photonics 1, 060801 (2016).

[5] A. Furusawa and P. van Loock, Quantum Teleportation and Entanglement, A Hybrid Approach to Optical Quantum Information Processing (Wiley, Weinheim, 2011).

[6] N. C. Menicucci, Fault-Tolerant Measurement-Based Quantum Computing with Continuous-Variable Cluster States, Phys. Rev. Lett. 112, 120504 (2014).

[7] B. Q. Baragiola, G. Pantaleoni, R. N. Alexander, A. Karanjai, and N. C. Menicucci, All-Gaussian Universality and Fault Tolerance with the Gottesman-Kitaev-Preskill Code, Phys. Rev. Lett. 123, 200502 (2019).

[8] K. Fukui, A. Tomita, A. Okamoto, and K. Fujii, High-Threshold Fault-Tolerant Quantum Computation with Analog Quantum Error Correction, Phys. Rev. X 8, 021054 (2018).

[9] K. Noh and C. Chamberland, Fault-tolerant bosonic quantum error correction with the surface-Gottesman-Kitaev-Preskill code, Phys. Rev. A 101, 012316 (2020).

[10] C. Flühmann, T. L. Nguyen, M. Marinelli, V. Negnevitsky, K. Mehta, and J. P. Home, Encoding a qubit in a trapped-ion mechanical oscillator, Nature (London) 566, 513 (2019)

[11] P. Campagne-Ibarcq, A. Eickbusch, S. Touzard, E. ZalysGeller, N. E. Frattini, V. V. Sivak, P. Reinhold, S. Puri, S.
Shankar, R. J. Schoelkopf, L. Frunzio, M. Mirrahimi, and M. H. Devoret, Quantum error correction of a qubit encoded in grid states of an oscillator, Nature 584, 368 (2020).

[12] D. Gottesman, A. Kitaev, and J. Preskill, Encoding a qubit in an oscillator, Phys. Rev. A 64, 012310 (2001).

[13] A. M. Steane, Active Stabilization, Quantum Computation, and Quantum State Synthesis, Phys. Rev. Lett. 78, 2252 (1997).

[14] S. Glancy and E. Knill, Error analysis for encoding a qubit in an oscillator, Phys. Rev. A 73, 012325 (2006).

[15] V. V. Albert, K. Noh, K. Duivenvoorden, D. J. Young, R. T. Brierley, P. Reinhold, C. Vuillot, L. Li, C. Shen, S. M. Girvin, B. M. Terhal, and L. Jiang, Performance and structure of singlemode bosonic codes, Phys. Rev. A 97, 032346 (2018).

[16] K. Noh, V. V. Albert, and L. Jiang, Quantum capacity bounds of Gaussian thermal loss channels and achievable rates with Gottesman-Kitaev-Preskill codes, IEEE Trans. Inf. Theory $\mathbf{6 5}$, 2563 (2019).

[17] G. Pantaleoni, B. Q. Baragiola, and N. C. Menicucci, Modular Bosonic Subsystem Codes, Phys. Rev. Lett. 125, 040501 (2020).

[18] C. Vuillot, H. Asasi, Y. Wang, L. P. Pryadko, and B. M. Terhal, Quantum error correction with the toric Gottesman-KitaevPreskill code, Phys. Rev. A 99, 032344 (2019).

[19] I. Tzitrin, J. E. Bourassa, N. C. Menicucci, and K. K. Sabapathy, Progress towards practical qubit computation using approximate Gottesman-Kitaev-Preskill codes, Phys. Rev. A 101, 032315 (2020).

[20] M. Gu, C. Weedbrook, N. C. Menicucci, T. C. Ralph, and P. van Loock, Quantum computing with continuous-variable clusters, Phys. Rev. A 79, 062318 (2009). 